\title{
Utilisation of controlled pore topology for the separation of bioparticles in a mixed-glass beads column
}

\author{
M. Mota*, J. Teixeira, A. Yelshin, S. Cortez \\ Centro de Eng. Biológica, University of Minho, Campus de Gualtar, 4710-057 Braga, Portugal \\ Received 27 January 2006; accepted 21 May 2006 \\ Available online 3 July 2006
}

\begin{abstract}
To study the flow of shaped particles in porous media, elution of spherical and rod-like micro-organisms was performed through beds of spherical glass beads. A $0.04 \mathrm{~cm} / \mathrm{s}$ constant flow rate was used with $5 \mu \mathrm{m}$ yeast suspensions, $1 \mu \mathrm{m}$ latex micro-spheres and rod-like bacilli Lactobacillus bulgaricus $6 \mu \mathrm{m}$ long and $0.5 \mu \mathrm{m}$ in diameter. Yeast cells' diameter is close to the bacilli length and micro-spheres have the same diameter as bacilli. All particle types have similar density. To make the different packing beds, $1.125 \mathrm{~mm}$ coarse beads and $0.1115 \mathrm{~mm}$ fine beads were used. Experiments were carried out using a column loaded with the binary packing (volume fraction of coarse particles in the mixture 0.7 ) or a monosize packing with the same amount of coarse or fine particles as used in the binary packing. Analysis of experimental results was based on two models: pure exclusion effect and hydrodynamic separation model [hydrodynamic chromatography (HDC)]. Results for spheres show that the classic HDC model fits to the experimental data whenever the ratio of particle size to the pathway bend scale is high $(\sim 1 / 100$, micro-spheres). However, if this ratio increases and becomes $\sim 1 / 20$, the HDC model needs to be corrected due to the effect of channel wall curvature on exclusion. This led to a modified HDC equation of the form $R=B /\left(1+2 \lambda-2.8 \lambda^{2}\right)$, where $R$ is the retention, $\lambda$ is the aspect ratio and constant $B \geq 1$. Bacillus separation follows an exclusion mechanism, since pore topology is important in the separation of shaped particles when the aspect ratio approaches $\lambda=0.1$. In the case of a binary packing bed, rod-like particles display a different behaviour than the one exhibited by the spherical particles of the same scale as bacilli, either in length or in diameter. This may be explained by the interaction between rod-like bacilli and the bed's pore topology. A generalised exclusion model for particles was proposed to be $R=A /(1-\lambda)^{z}$, where $A$ is the coefficient proportional to the tortuosity and the parameter $z=1$, 2 or 3 depends mainly on pore shape. Controlled pore topology opens interesting applications for bio-separation (in porous micro-fluidic devices, deep bed filtration) and might be especially important for macromolecules and micro-organisms separation with different shapes.
\end{abstract}

(C) 2006 Elsevier B.V. All rights reserved.

Keywords: Porous media; Flow; Particle shape; Separation; Models; Pore topology

\section{Introduction}

Motion of colloids and micro-organisms in porous media is a current phenomenon in nature and industry [1-6] and it is important to know how particle size and shape affect a particulate dispersion during flow through tortuous porous media.

A list of effects related with the pore topology and particle shape accompany the flow of micro-particles in a porous medium, namely, the ratio of particle size to the pore size or hindrance effect, constraints brought about by abrupt changes in pore cross-section area, and, among others, channel tortuosity.

\footnotetext{
* Corresponding author. Fax: +351 253678986.

E-mail address: MMota@ reitoria.uminho.pt (M. Mota).
}

In general, the tortuosity factor is a combination of both the average pathway tortuosity of micro-particles and the shape factor (constrictions) of the pore channel. It is plausible to speculate that the tortuous pathway, at appropriate conditions, may create a disorder momentum for rod-like objects between the flow trajectory and the main particle axis direction. The disorder momentum reduces the rod-like micro-particle flow velocity as compared with the flow of spherical particles of similar weight. The tortuosity effect on rod-like particles motion in porous media will be analysed in the following sections.

\section{Theoretical background}

Micro-particle separation, under non-equilibrium conditions, of a dispersion flowing through porous media may be performed by hydrodynamic chromatography (HDC) based upon 
a void excluding effect and by slalom chromatography (SC) related with a hindrance effect generated in the tortuous channels.

Hydrodynamic chromatography, as a size separation technique, is generally applied in two ways: using capillaries or using columns packed with monosize non-porous particles in the size range 1-1.5 $\mu \mathrm{m}$ and 10-20 $\mu \mathrm{m}$ [7-9]. It must be underlined that most of HDC theories have been derived by modelling the flow of the dispersed phase in open straight capillaries [10-13], where there is no tortuosity. The dynamic range of the separation depends on the particle size of the packing media in the column and on separating micro-particles in the dispersed phase [14,15].

For macromolecules and micro-particles, the diffusion coefficient is small and longitudinal diffusion is negligible compared to the effect of convective mixing. At high solvent velocity, this fact can be written in the form of plate height as $H=1.4 d_{\mathrm{p}}$, where $H$ is independent of the solvent's velocity [7-9,16,17]. In this way, a bundle of capillary tubes can be used as a model for the migration rate of solutes in a packed column. However, this model ignores totally the effect of tortuosity, which, as is known, is more significant at very small scales [18].

The migration of macromolecules or micro-particles is characterised by a retention value $R=t_{\mathrm{m}} / t_{0}$ versus an aspect ratio $\lambda=d_{\mathrm{e}} / d_{\text {por }}$ in the form adopted by Venema et al. and Blom et al. $[9,19]$ :

$R=\frac{B}{1+2 \lambda-C \lambda^{2}}$

where $B=1.0 ; t_{\mathrm{m}}$ and $t_{0}$ are the migration time of micro-particle and of an infinitely small sized marker, respectively; $C$ is a coefficient described below, $d_{\mathrm{e}}$ is the micro-particle effective diameter; and $d_{\text {por }}$ is the diameter of the capillary or pore. The effective pore diameter $d_{\text {por }}$ for the granular packing is equal to

$d_{\mathrm{por}}=\left(\frac{2}{3}\right) d_{\mathrm{p}} \frac{\varepsilon}{1-\varepsilon}$

where $\varepsilon$ and $d_{\mathrm{p}}$ are, respectively, the column packing porosity and particle size.

Coefficient $C$ in Eq. (1) is a constant accounting for secondary effects and ranges from 0.5 to $5[9,19]$. For the simple exclusion effect in a parabolic flow profile, $C=0.5$ [19]. In a packed particulate bed, recommended values of $C$ are in the range $C=2.698-2.8[9,15,20]$.

The correct estimate of the effective molecule size is important in the case of macromolecules due to their flexibility or shape. The flow rate dependence of macromolecules of different sizes can be explained in terms of deformation and orientation of simple macromolecular models in steady uniaxial elongation [21]. The importance of the flow can often be estimated with the Deborah number, $D e$, i.e., a ratio of the hydrodynamic forces to the Brownian forces. Significant molecular stretching in steady flows occurs only for $D e>0.5$.

Contrary to flexible macromolecules, rigid rod-like macromolecules are able to orient their axis in strong flow and during flow in the small tortuous channels of the column can also yield a flow-dependent elution behaviour [12].
Other investigations show that for rigid and semi-flexible macromolecules, HDC can shift at some region of $\lambda$ to the separation mechanism called the slalom chromatography (SC) [22]. $\mathrm{SC}$ is based on the effect on macromolecules motion across a porous medium caused by channel tortuosity in the regime of "snake-like", reptation or slalom-like manner [23-29].

Theoretical background of SC is still giving the first steps and the transient condition between HDC and SC is under investigation. In both cases (HDC and SC), the effect of tortuosity and pore topology separation is insufficiently known.

Investigation of passive, point-size, Brownian solute particles motion demonstrates that a serpentine geometry is equivalent to a straight channel, when the channel's tortuosity is taken into account [30,31]. However, additional effects causing distortion of the velocity profile cannot be explained by this simplified approach.

Particles flow through a porous medium is accompanied by effects related with pore topology and particle shape. In general, the tortuosity factor derives from a combination of both the tortuosity of the pathway and the pore channel's shape.

Unlike sphere-like micro-particles, rod-like and linear species undergoing separation behave differently even when other physical-chemical effects are omitted. The tortuous pathway, especially in the form of bends, may create, in appropriate conditions, a disorder momentum on the rod-like object. This fact may reduce the rod-like micro-particle flow velocity as compared with the flow of spherical particles of similar weight, thereby yielding a retardation effect. In Fig. 1, the schematic motion of a rod-like particle in a tortuous channel is shown.

The disturbing factor brought about by the motion of rod-like particle across a bend is caused by the change of the particle axial orientation relative to the flow streamlines. In the bends, the streamlines and the main particle axis are divergent: rod-like particles expose a higher cross-section area thereby increasing the friction force and yielding retardation [32].

A rod particle relaxation effect in fluid flow may be characterised by the Deborah number, $D e$, that can be represented as

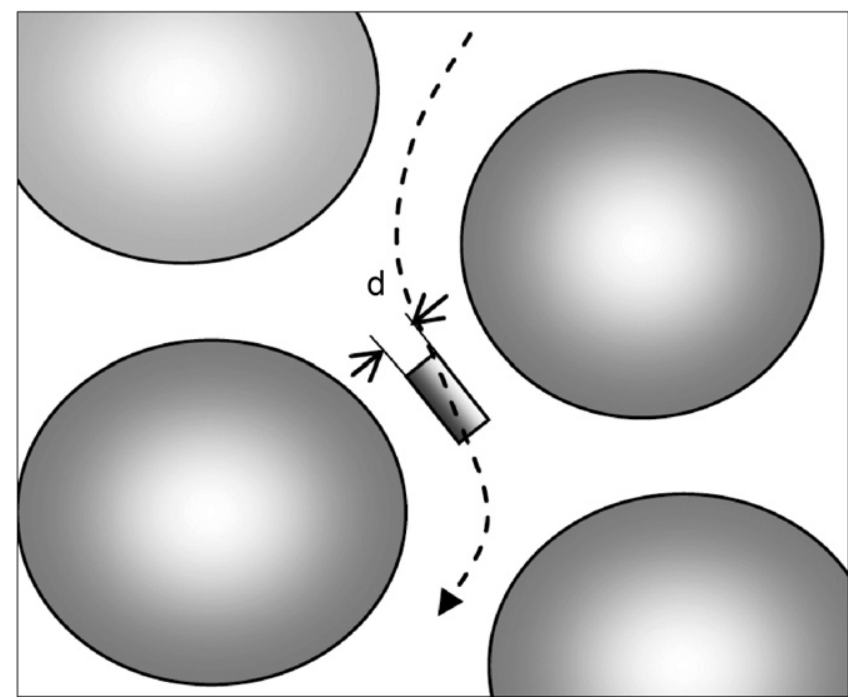

Fig. 1. 
the ratio of a moving relaxation time $\theta$ to the time to which the object was exposed to deformation $\theta_{\mathrm{p}}$ [33]

$D e=\frac{\theta}{\theta_{\mathrm{p}}}$

where $\theta_{\mathrm{p}}$ is determined by porous media properties: $\theta_{\mathrm{p}}=a \varepsilon d_{\mathrm{p}} / u$, where $\varepsilon$ is the bed porosity, $d_{\mathrm{p}}$ is the particle diameter, and $a$ is a numerical coefficient. When $a$ in $\theta_{\mathrm{p}}$ is assumed to be 1.0 , the Deborah number becomes $D e=u \theta / \varepsilon d_{\mathrm{p}}$.

For rod-like particles the estimation of relaxation time, $\theta$, is important and can be made using an analogy between flexible and rigid macromolecules.

\subsection{Rod-like micro-particles motion in tortuous channels}

Let us assume that the packing is made up with spherical particles of size $d_{\mathrm{p}}$ and that the tortuosity of pore channels affects the micro-particle flow exclusively by the number of bends (Fig. 1), i.e., an increase in the number of bends increases retardation. Therefore, we can estimate the number of bends, as, for a defined packing, the number of bends will be proportional to the particle size $d_{\mathrm{p}}$.

Other parameter linked with the micro-particle is the ratio $\lambda$ of a micro-particle "effective" size $d_{\mathrm{e}}$ to the pore size $d_{\text {por }}$. The effective size for rods lies within the range of minimum and maximum geometrical scales (diameter and length) and depends on flow velocity.

The contribution of the bend number to pore topology can be presented as follows. If $h$ is the bed thickness and the tortuosity $T$ of the average pathway of length $L_{\mathrm{e}}$ is $T=L_{\mathrm{e}} / h$, then the number of bends $n_{\mathrm{b}}$ is $n_{\mathrm{b}}=L_{\mathrm{e}} / d_{\mathrm{p}}=T h / d_{\mathrm{p}}$. For example, in a monosized coarse sphere packing of $h=0.3 \mathrm{~m}$ and $d_{\mathrm{p}}=1125 \mu \mathrm{m}$, the number of bends is $n_{\mathrm{b}}=373$ assuming a tortuosity $T=1.4$. This implies that we shall have $373 / 0.3=1243$ bends per metre of bed height.

The number of bends can be increased significantly using a mixed particle bed [34]. For a binary mixture of particles, the overall packing tortuosity $T$ becomes a product of micro- and macro-scale tortuosity $T_{\mathrm{f}}$ and $T_{\mathrm{c}}$, respectively [35-37]: $T=T_{\mathrm{f}} T_{\mathrm{c}}$. Consequently, number of bends for the binary packing can be written as

$n_{\mathrm{b}}=T \frac{h}{d_{\mathrm{p}}}=T_{\mathrm{f}} T_{\mathrm{c}} \frac{h}{d_{\mathrm{f}}}$

where $d_{\mathrm{f}}=d_{\mathrm{p}}$ is the diameter of fine spheres in the packing.

Macro-scale tortuosity $T_{\mathrm{c}}$ (coarse particle fraction of size $d_{\mathrm{c}}$ ) increases the pathway length per column, whereas the microscale tortuosity $T_{\mathrm{f}}$ (fine particle fraction of size $d_{\mathrm{f}}$ ) accounts for the number of bends per bed thickness. Now, according to Eq. (4), for $h=0.3 \mathrm{~m}$ and $d_{\mathrm{f}}=111.5 \mu \mathrm{m}$, the bend number may be written as $n_{\mathrm{b}}=2.7 \times 10^{3} T$. For large coarse particle size ratio $\left(d_{\mathrm{c}} / d_{\mathrm{f}} \geq 10\right)$ it is possible to assume for a binary sphere packing $T_{\mathrm{f}}=T_{\mathrm{c}} \sim 1.45$ and finally the overall packing tortuosity becomes $T=(1.45)^{2}=2.1$, i.e., $n_{\mathrm{b} \text { (binary) }}=2.7 \times 10^{3}$; $2.1 / 0.3=18,900$ bends $/ \mathrm{m}$. This value will be 1.45 times higher than the one obtained for pure fine particles packing with the same bed thickness and 15 times higher than the bend number for the coarse particle packing with the same bed height. Furthermore, we are working at the micro-scale, since the dimension of a single bend will be of the order of $1 / 18,900 \mathrm{~m}=5.3 \times 10^{-5} \mathrm{~m}$. From the estimation made, it is thus possible to expect a higher retardation with the binary packing for rod-like micro-particles. This expectation will be assessed experimentally as will be described in the following sections.

\section{Materials and methods}

\subsection{Micro-particles}

To build a column packing, it is necessary, at first, to choose micro-particles for experiments. As experimental models, Lactobacillus bulgaricus of length $\sim 5-6 \mu \mathrm{m}$ and diameter $\sim 0.5 \mu \mathrm{m}$ was used to represent rod-like particles. Their flow behaviour through porous media was compared with the one observed for yeast cells (Saccharomyces cerevisiae, size $\sim 5 \mu \mathrm{m}$ ) and with $1 \mu \mathrm{m}$ spheres. Both yeast and spheres have one dimension similar to the bacillus (Fig. 2).

The dispersed phase flow was investigated for three types of bed packing: fine particles, coarse particles, and a mixture of fine and coarse particles.

\subsection{Binary packing and column characteristics}

Particles of dispersed phase and mixed bed packing were chosen so that the ratio of pore size to the dispersed particles was in the range recommended for HDC $[9,38]$ as well as in the range of pore topology sensitivity defined by $2 \mathrm{D}$ fractal analysis. Assuming that the dispersed particle size ratio of $d_{\mathrm{e}}$ to the pore size $d_{\text {por }}$ must be $\lambda=d_{\mathrm{e}} / d_{\text {por }} \sim 0.01$, then, for fine packing, at $d_{\mathrm{e}}=5 \mu \mathrm{m}$, the bed particle size should be around $0.1 \mathrm{~mm}$. Therefore, glass beads of size $d_{\mathrm{f}}=0.1115 \mathrm{~mm}$ were chosen as the fine particles.

Mixed bed packing characteristics and coarse particle size were selected by means of binary packing analysis at different

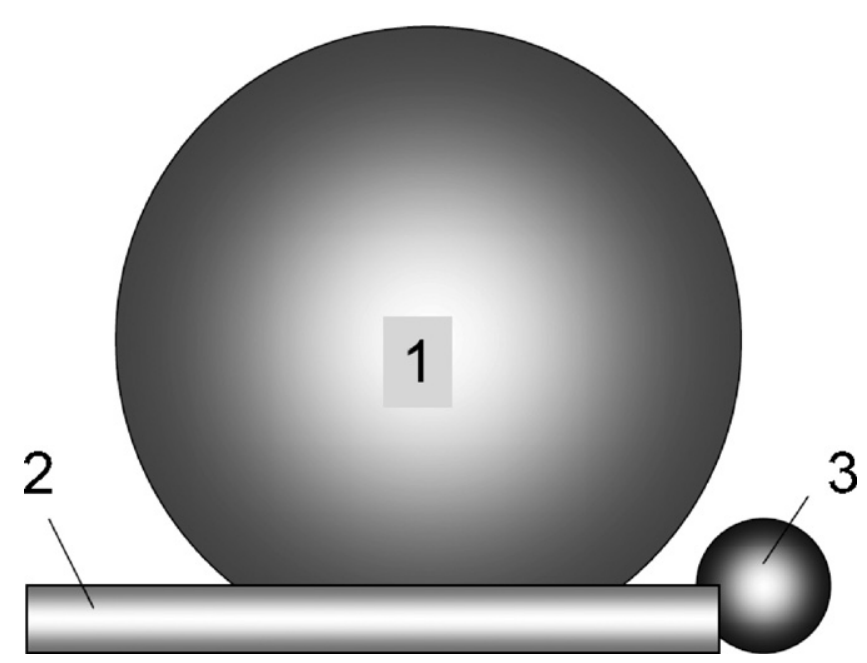

Fig. 2. 


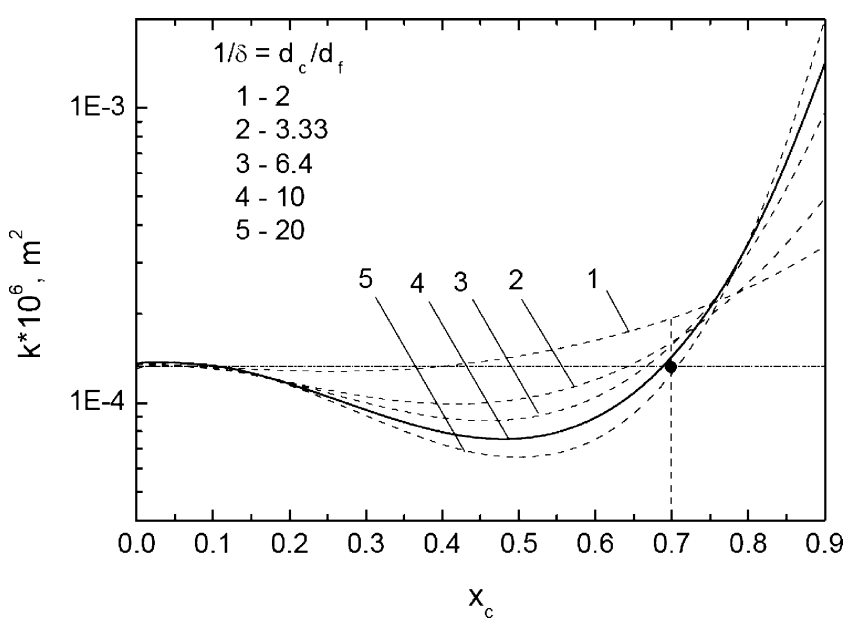

Fig. 3.

fine/coarse particle size ratio $\delta=d_{\mathrm{f}} / d_{\mathrm{c}}$. The same approach was used to select the coarse particles' fractional content $x_{\mathrm{c}}$, where $x_{\mathrm{c}}$ is the fraction of coarse particles in the mixture: $x_{\mathrm{c}}=$ (volume of coarse particles)/(volume of coarse + fine particles). The analysis was performed using the binary packing model described in a work of Mota et al. [37].

To obtain the maximum effect of the bend number, the binary packing must be in the range of minimum porosity $\varepsilon_{\min }$ that corresponds to $x_{\mathrm{c}} \sim 0.7$. Furthermore, an additional condition was imposed: the permeability of fine and binary packing must be close to each other.

The packing permeability $k$ simulated by the model [37] is shown in Fig. 3, where the porosity of fine and coarse packing was assumed to be $\varepsilon_{0}=0.37$.

Binary packing of $\delta=d_{\mathrm{f}} / d_{\mathrm{c}} \sim 0.1$ (curve 4 , Fig. 3) fulfils the imposed condition. Its permeability is close to the fine particles packing at $x_{\mathrm{c}}=0.7$, and glass beads of size $d_{\mathrm{c}}=1.125 \mathrm{~mm}$ were chosen as coarse particle fraction.

The simulation gives 0.22 for the binary packing porosity $\varepsilon$, curve 1 of Fig. 4, but even so, the permeability at $x_{\mathrm{c}}=0.7$ is close to the one of the fine packing because of the higher pore size $d_{\text {por }}$

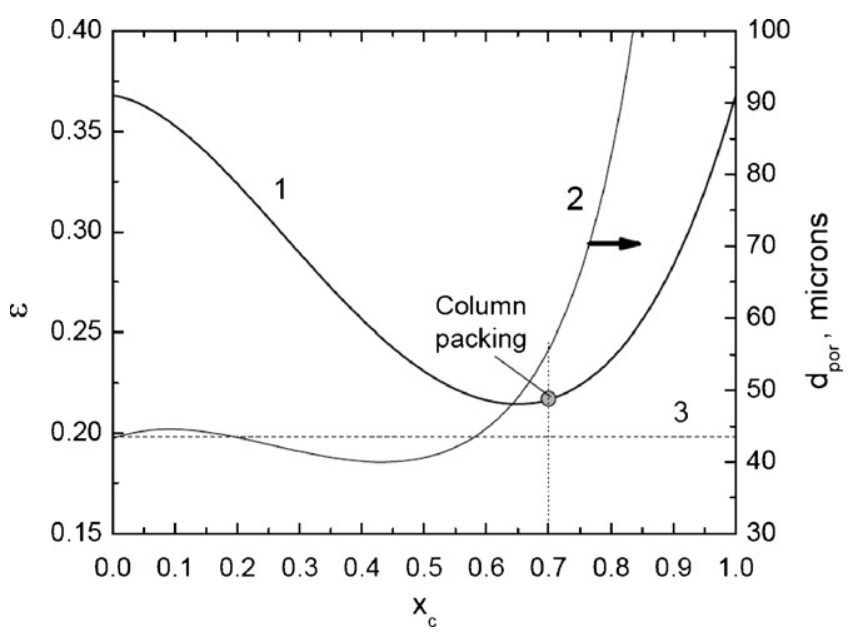

Fig. 4.
(Fig. 4, curve 2). For the dependence $d_{\text {por }}$ versus $x_{\mathrm{c}}$, Eq. (2) was used, with $d_{\mathrm{p}}=d_{\mathrm{av}}$, where $d_{\mathrm{av}}=1 /\left[x_{\mathrm{c}} / d_{\mathrm{c}}+\left(1-x_{\mathrm{c}}\right) / d_{\mathrm{f}}\right]$ is the average particle size of the binary packing of coarse $\left(d_{\mathrm{c}}=1.125 \mathrm{~mm}\right)$ and fine $\left(d_{\mathrm{f}}=0.1115 \mathrm{~mm}\right)$ particles. The binary packing pore tortuosity was $T=1 / \varepsilon^{0.4} \approx 1.83$ against $T=1.49$ calculated for the fine packing.

\subsection{Packing materials}

From the estimations presented earlier, two types of silica glass beads with homogenous and smooth surface, high roundness (ratio of axis $\geq 95 \%$ ) and narrow size ranges were used in experiments for the column packing: coarse particles from Sigmund Lindner, type $S$ with average diameter $d_{\mathrm{c}}=1.125 \mathrm{~mm}$, and fine particles - micro-spheres from Sovitec, type AF with average diameter $d_{\mathrm{f}}=0.1115 \mathrm{~mm}$. Particle size deviation from average is $\pm 15 \%$. A transparent column, in acrylic, with $42 \mathrm{~mm}$ of inner diameter was used in all experiments.

Experiments were made with a binary packing column at $x_{\mathrm{c}}=0.7$ as well as with columns made exclusively of coarse and fine particles in the same amount of coarse or fine particles as used in the binary bed. Dextran blue was used for checking the column packing checking and also as a tracer [39].

The mixed bed was prepared using an aqueous solution of glycerol to avoid particles segregation. The detailed procedure is described in the work of Dias et al. [40]. The procedure includes mixing the glass beads in the appropriate proportion with a glycerol aqueous solution, filling the column, packing the column and washing the glycerol out. The binary packing height was $\sim 15 \mathrm{~cm}$. Packing porosity was determined experimentally by a volumetric method.

Sterile buffers were pumped to the column using a peristaltic pump. Approximately 3-4 bed volumes of sterile buffer solution with the same $\mathrm{pH}$ and ionic strength as the microbial solution were passed through the column at the start-up of each experiment.

\subsection{Dispersed phase}

\subsubsection{Bacterium}

The bacterium culture used in this study was L. bulgaricus, a long rod-like bacillus with approximately $6.80 \pm$ $2 \mu \mathrm{m} \times 0.54 \mu \mathrm{m}$ from the Belgian bacteria collection BCCM/LMG. Growth was done in slants containing MRS agar at $37^{\circ} \mathrm{C}$, under reduced oxygen tension.

\subsubsection{Yeast}

A strain of $S$. cerevisiae was used. Cells were obtained from baker's yeast that was inoculated in plates containing yeast extract peptone dextrose (YEPD) at $30^{\circ} \mathrm{C}$. Yeast cells have a spheroid shape with a mean diameter of around $5 \pm 1 \mu \mathrm{m}$.

Before filtration, cells of each strain were grown separately. To avoid the agglomeration of cells after growth, $100 \mathrm{~mL}$ of cells of each culture were treated by ultrasound. The same volume of $L$. bulgaricus and $S$. cerevisiae culture was then centrifuged at $12,000 \mathrm{rpm}$, at $4{ }^{\circ} \mathrm{C}$ for $10 \mathrm{~min}$. The resulting 


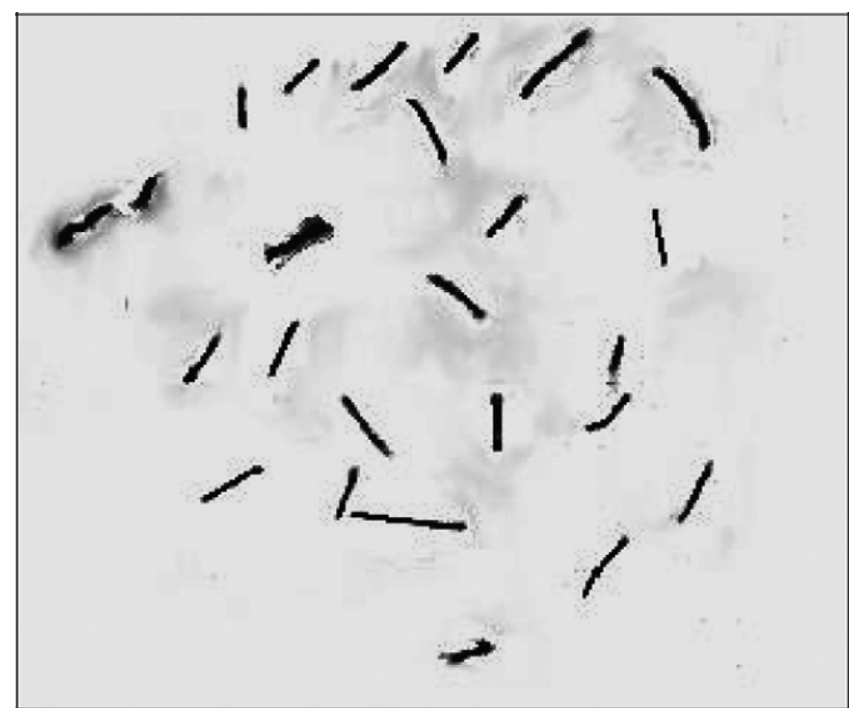

(a)

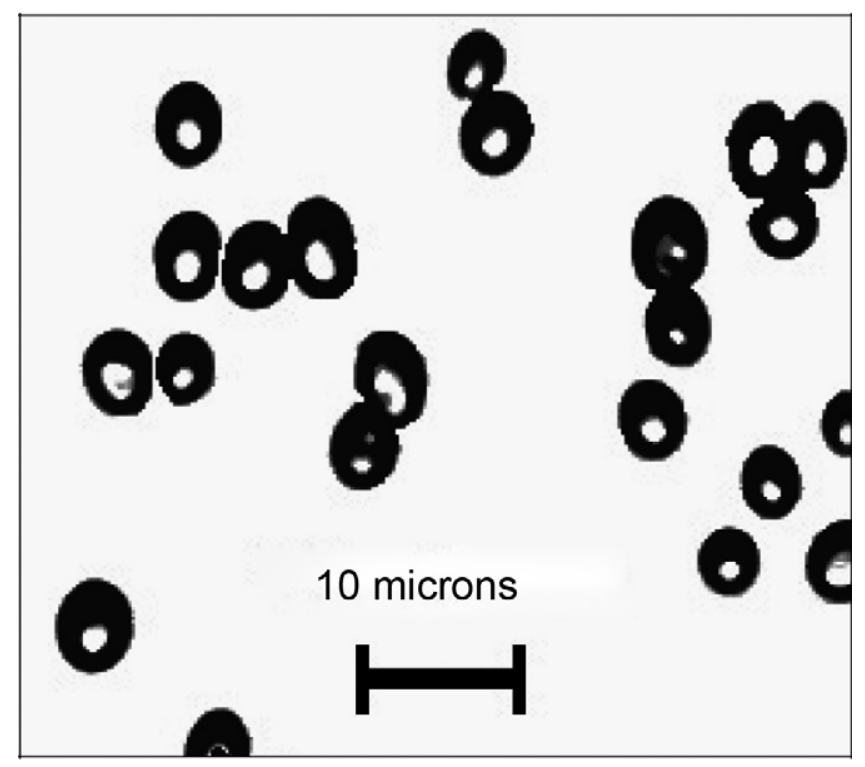

(b)

Fig. 5.

pellet was re-suspended in sterile buffer solution (PBS pH 7.2 and $\mathrm{NaCl} 8 \mathrm{~g} / \mathrm{L}$ ). This procedure was repeated three times. Finally, formalin $4 \%(\mathrm{v} / \mathrm{v})$ was added to the re-suspended cells.

Bacterial and yeast sizes and cell concentration were estimated via image analysis. Images were captured in a microscope Leitz with the program AxioVision 3.1. A typical view of microorganisms is shown in Fig. 5.

The microbial solutions used on filtration tests had a cell concentration of the order of $2.80 \times 10^{6}$ cells $/ \mathrm{mL}$.

Monodisperse green fluorescent polymer micro-spheres (aqueous) were supplied by Duke Scientific Corporation, with a polymer density of $1.05 \mathrm{~g} / \mathrm{cm}^{3}$ and a concentration of $1.8 \times 10^{10}$ micro-spheres $/ \mathrm{mL}$. Their average size was $1 \mu \mathrm{m}$.

All particle types - micro-spheres and micro-organisms - had similar density $\sim 1.05-1.1 \mathrm{~g} / \mathrm{cm}^{3}$.

\subsection{Experiments}

Elution was performed at a constant apparent flow velocity at binary packing of $0.04 \mathrm{~cm} / \mathrm{s}$ [41]. The main measured parameters were particle breakthrough curves, retention coefficient $R=t_{\mathrm{m}} / t_{0}$, and ratio of particle size to the pore size $\lambda$, where $t_{\mathrm{m}}$ and $t_{0}$ are, respectively, the elution time of the sample particles and the tracer. Each test was performed four to six times. In the end of each experiment, 30 glass beads were collected from the column and inspected microscopically to check for adhered cells or particles.

\section{Results}

Typical results obtained are given below in the form of the normalised concentration $C_{\mathrm{n}}$, a ratio of eluted concentration to the maximum measured eluted concentration. Micro-particles separation on the binary packing column is shown in Fig. 6 . The retention time was calculated based on the equation $R=$ $t_{\mathrm{m}} / t_{0}=\left(v_{\mathrm{m}} / u\right) /\left(v_{0} / u\right)=v_{\mathrm{m}} / v_{0}$, at constant $u$, where $v_{\mathrm{m}}$ and $v_{0}$ are, respectively, the elution volumes of the sample microparticles and the tracer.

Fig. 6 shows that in the mixed column, spherical microparticles of different sizes (micro-spheres and yeast) do not follow the HDC law, which leads to the hypothesis that hindrance effect is present. For rod-like bacillus the retardation effect is more pronounced. Peaks shape is close to the Gaussian and allows assuming that adsorption effect is negligible, since no cells or particles were observed microscopically in the collected samples of glass beads.

Breakthrough curves for monosize packing of fine and coarse particles are shown in Figs. 7 and 8. In monosized cases, the amount of fine and coarse particles was the same as in the previous binary packing. Due to the small pore size in the fine particle packing, around $43 \mu \mathrm{m}$, only Dextran and micro-spheres were able to cross the column (Fig. 7). Peak asymmetry in Fig. 7 is explained by packing quality that was recognised as a loose packing.

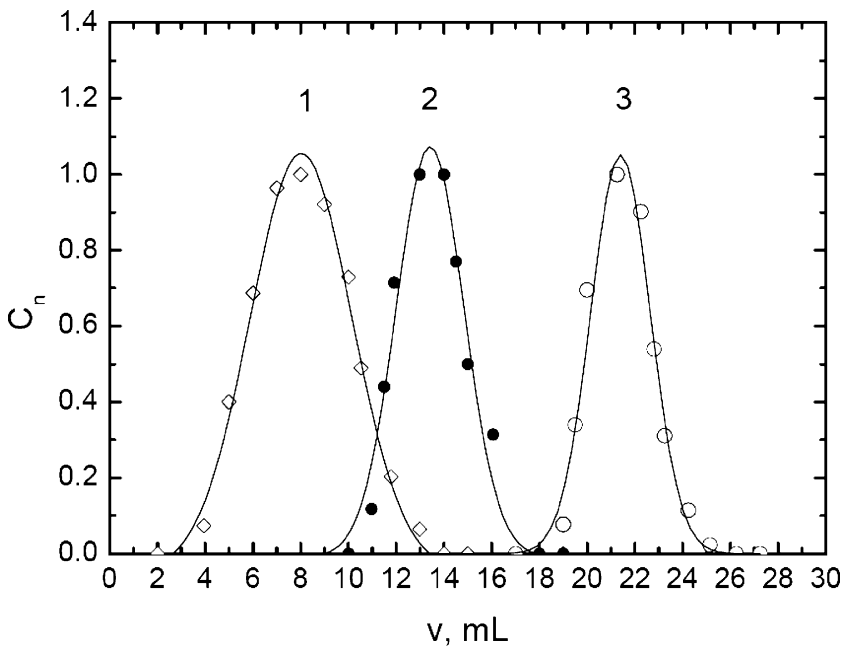

Fig. 6. 


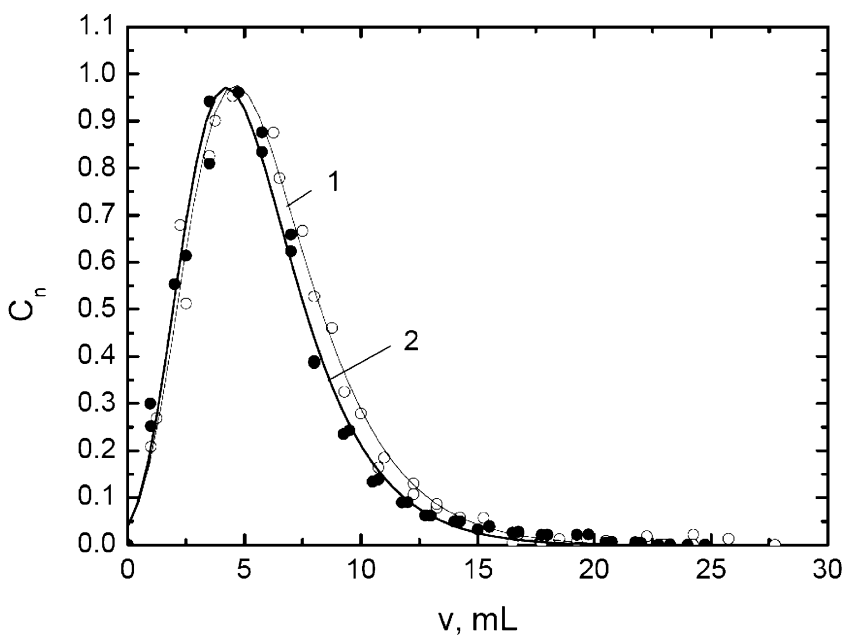

Fig. 7.

In the work of Cernanský [42], a geometrical construction inscribed in the pore allowed to estimate the critical particle size able to cross a monosize packing of spheres: $d=d_{\mathrm{p}}\left\{k_{\mathrm{g}}(\pi /(1-\varepsilon))^{1 / 3}-1\right\}$, where, depending on the packing structure $(2 / \sqrt{3})(\sqrt{2} / 6)^{1 / 3} \leq k_{\mathrm{g}} \leq(\sqrt{2} / 3)^{1 / 3}$, that for fine packing gives $d=0.137$ and $d_{\mathrm{p}}=25 \mu \mathrm{m}$. According to Ippolito et al. [43] the diameter ratio of spheres moving in a bed to the packing particles diameter has the critical value of 0.1547. Above this critical ratio, beads get imprisoned inside the packing. In practice, due to physical-chemical effects, the critical particle size is even smaller. According to Beverly [44], a granular filter media will generally remove particles $7-10 \%$ larger than the media grains. In the case of the present work, this corresponds to micro-particles $8-11 \mu \mathrm{m}$ in size, which is close to the main size of yeasts and bacilli.

In the present work, the fraction of each particle able to cross the fine column substrate was Dextran blue, $100 \%$; microspheres, 79.2\%; S. cerevisiae and L. bulgaricus, $0 \%$.

Insignificant HDC effect was observed for micro-spheres in comparison with Dextran both in fine and coarse beds (Figs. 7 and 8).

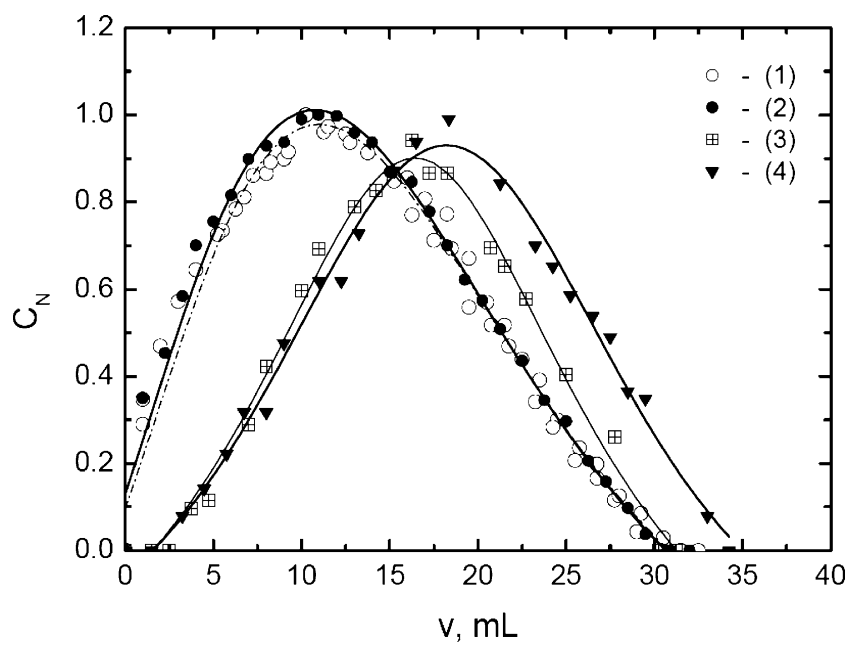

Fig. 8.
Table 1

Characteristics obtained during experiments on different columns

\begin{tabular}{llll}
\hline Parameter & Fine & Mixed bed & Coarse \\
\hline Particle size $(\mu \mathrm{m})$ & 111.5 & 300 & 1125 \\
Porosity & 0.381 & $0.217-0.228$ & 0.385 \\
Pore size, $d_{\text {por }}(\mu \mathrm{m})$ & $\sim 43.3$ & 59 at $\varepsilon=0.228$ & 470 \\
$V_{\text {total }} / V_{0}=1 / \varepsilon$ & 2.62 & $4.4-4.6$ & 2.6 \\
Flow velocity, $u / \varepsilon(\mathrm{cm} / \mathrm{s})$ & 0.105 & 0.175 & 0.104 \\
$\lambda$ & & & \\
$\quad$ & & & 0.002 \\
$\quad$ Micro-spheres & 0.023 & 0.017 & \\
$\quad$ Bacillus & & & 0.013 \\
$\quad$ By length $6 \mu \mathrm{m}$ & 0.139 & 0.1 & 0.001 \\
$\quad$ By diameter & 0.012 & 0.0085 & 0.011 \\
$\quad$ Yeast (size $5 \mu \mathrm{m})$ & 0.115 & 0.085 & 1 \\
Appearance & & & $\sim 2$ \\
$\quad$ Micro-spheres & 1 & 1 & $\sim 3$ \\
$\quad$ Bacillus & - & 3 & \\
$\quad$ Yeast & - & 2 & \\
\hline
\end{tabular}

Separation of yeasts and bacilli in a coarse bed shows, as with the mixed column, that yeast cells emerge later than microspheres whereas the retardation effect of bacillus is not as pronounced. The observed results indicate that, together with the aspect ratio $\lambda$, other factors, such as tortuosity and non-HDC conditions, may affect separation. The obtained data are summarised in Table 1.

Kraak $[9,38]$ recommended for HDC an aspect ratio $\lambda>0.01$. In their work, Peyrin et al. [25] admitted that the separation in HDC occurs when the ratio $\lambda$ is between 0.01 and 0.35 , but this condition is only valid when the polymer is in a random coil form. However, with $\lambda$ around 0.1 , the random coil deformation was observed even for low flow velocity. Using the available HDC results on latex particles [45-49], it was possible to define boundaries for HDC separation: the upper boundary corresponds to $\lambda=0.05-0.07$ and the lower boundary to $\lambda=0.002-0.007$, depending on the packing particle size.

Summarising this observation and the obtained data (Table 1), it is plausible that HDC conditions are not valid for all tested micro-particles. Nevertheless, as was mentioned earlier, the expected value of $\lambda$ for the bend approach is in the range of $0.08>\lambda>0.01$.

\section{Discussion}

Dependences of micro-particles retention $R$ on the aspect ratio $\lambda$ for various column packings are given in Fig. 9, where points marked by thick arrows belong to the coarse particle packing.

\subsection{Micro-spheres}

For micro-spheres in all packing types, the aspect ratio is higher than 0.025 and their behaviour corresponds to HDC at binary packing $(\lambda=0.017)$ with $R=0.88$ against the Dextran blue macromolecules but less pronounced at $\lambda=0.002$, with coarse packing (Fig. 8) because at this value the HDC lower 


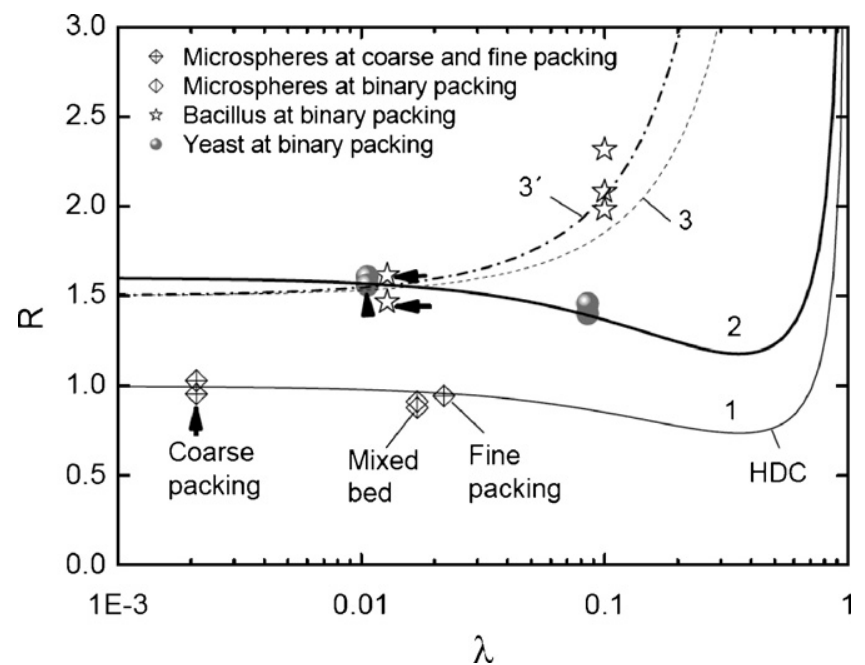

Fig. 9.

boundary is attained. The obtained $R$ at different beds fits with HDC model (1) at $C=0.28$ (Fig. 9, curve 1).

\subsection{Yeast}

Yeast spheroid cells of $S$. cerevisiae behave differently from micro-spheres because the aspect ratio $\lambda$ is close to the upper HDC bound. Anyway, as shown in Fig. 9, experimental points for binary and coarse packings are simulated by the HDC model (1) with displacement $B=1.6: R=1.6 /\left(1+2 \lambda-2.8 \lambda^{2}\right)$.

Results for spheres show that the classic HDC model $(B=1.0)$ is fitting well to the data whenever the ratio of particle size to the bend scale (fine particles fraction) is high $\sim 1 / 100$ and particles are inside the Brownian scale (micro-spheres). However, if the micro-particles size increases (yeast), the HDC model needs to be corrected because of decreasing particles spatial "mobility" = hindrance and to the effect of channel wall curvature on exclusion by the coefficient $B>1.0$. We may speculate that the gravitational force can also affect the cells trajectory.

Let us compare the obtained results with the hindered diffusion model.

In the case of hindered diffusion, the effective diffusion coefficient of a solute in the channel $D_{\mathrm{e}}$ is composed of two correction functions $F_{1}(\lambda)$ and $F_{2}(\lambda)[50]$ :

$D_{\mathrm{e}}=D_{0} F_{1}(\lambda) F_{2}(\lambda)$

where $D_{0}$ is the bulk diffusion coefficient. The parameter $F_{1}(\lambda)$ is the steric partition coefficient, which is defined as the crosssectional area of the pore available to the solute molecule divided by the total cross-sectional area of the pore and defined by equation $F_{1}(\lambda)=(1-\lambda)^{2}$.

The correction factor $F_{2}(\lambda)$ accounts for the effect of the pore wall on the solvent properties (an increase in the local solvent viscosity near the pore wall) and is often represented by a polynomial series or an exponential function.

Usually, restricted diffusion models are assuming smooth cylindrical pores even when the solute passage is tortuous [51].
Transition to the tortuous channel leads to the expression

$D_{\mathrm{e}}=D_{0} \frac{1}{\tau} F_{1}(\lambda) F_{2}(\lambda)$

where $\tau$ is the tortuosity factor. Hence, if the channel represents a linear cylinder then $\tau=1.0$. In the context of the retardation effect, $R \propto D_{0} / D_{\mathrm{e}}$, Eq. (6) becomes

$R=\frac{\tau}{F_{1}(\lambda) F_{2}(\lambda)}$

The effective diffusivity (5) is well described by Renkin equation [52], where $F_{2}(\lambda)$ is expressed as:

$F_{2}(\lambda)=1-2.1 \lambda+2.1 \lambda^{3}-0.95 \lambda^{5}$

The dependence (7) of $R$ on $\lambda$ for the pure exclusion effect, $F_{2}(\lambda)=0$, and the Renkin Eq. (8) are shown in Fig. 10.

From Fig. 10 it follows that the simple exclusion model, represented by curves 1 and $1^{\prime}$, is not fitting, whereas the Renkin approach fits to some experimental values but cannot simulate the whole yeast experimental data set. The HDC model can be modified by introducing a hindrance parameter related with the tortuosity factor, which, in this case, may be achieved by making the coefficient $B>1.0$. By taking $B=1.6$ yeast results fit reasonably to the HDC model, for $\lambda>0.01$ (Fig. 9).

We may speculate that the pore topology has a geometrical effect on particles. Two-dimension simulations, mentioned in Section 2, were made with mono and binary packing of disks. Pore area fractal analysis was performed by a test-box method where the box simulates a compact particle inside the pore.

The measured parameter was the fractal dimension of pore area $D_{\mathrm{F}}$ versus aspect ratio $\lambda^{\prime}$. This aspect ratio is the ratio between the test-box size and the pore size. Sensitivity estimations of $D_{\mathrm{F}}$ to $\lambda^{\prime}$ were done. For a hexagonal monosize $2 \mathrm{D}$ packing, the simulation gave for $\lambda^{\prime} \sim 0.01, D_{\mathrm{F}} \sim 1.8$; for $\lambda^{\prime} \sim 0.05, D_{\mathrm{F}} \sim 1.4$; and for $\lambda^{\prime}=0.1, D_{\mathrm{F}} \sim 1.1 / 1.2$.

Simulation of a binary $2 \mathrm{D}$ packing with particle size ratio $D / d=15$ yielded more complex results since the fractal dimension becomes dependent on the binary composition content. For

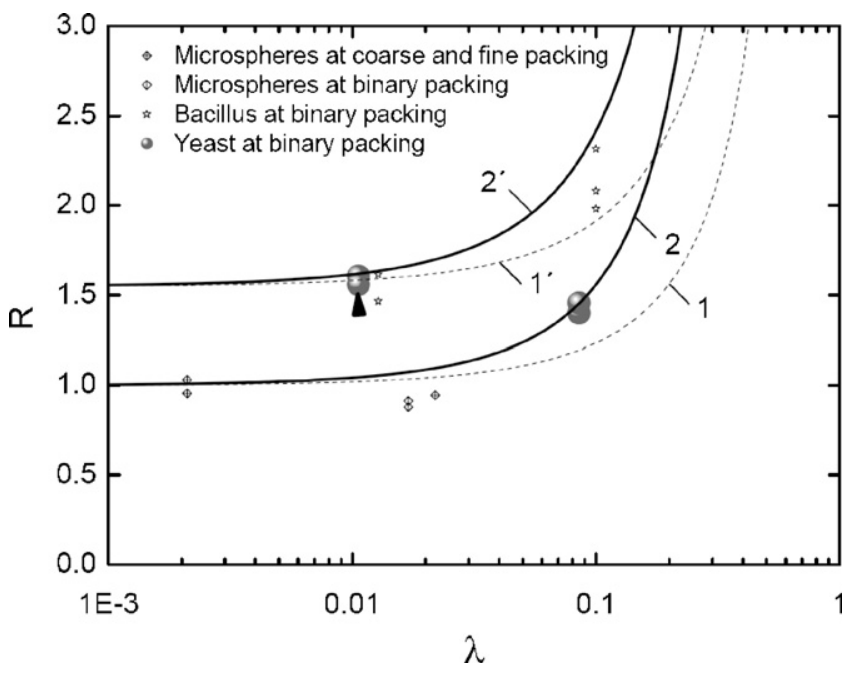

Fig. 10. 
instance, monosize coarse and fine packings of discs used in simulated binary structures gave for $\lambda^{\prime} \sim 0.01, D_{\mathrm{F}} \sim 1.7$ and for $\lambda^{\prime} \sim 0.1, D_{\mathrm{F}} \sim 1.3$, respectively. A drastic reduction of $D_{\mathrm{F}}$ was observed when the pure coarse packing was replaced with a binary mixture with minimum porosity from $D_{\mathrm{F}} \sim 1.7$ up to 1.5 . A further linear decreasing was noticed toward the pure fine packing (up to $D_{\mathrm{F}} \sim 1.3$ ) despite the aspect ratio in the region of minimum porosity $\lambda^{\prime} \sim 0.08$ not being far from $\lambda^{\prime} \sim 0.1$ for pure fine packing.

In the binary packing, a change in 2D fractal dimension from 2.0 toward 1.0 can be, in 3D space, considered by analogy equivalent to a reduction of $D_{\mathrm{F}}$ from 3.0 to 2.0 or less. This provides an explanation of the yeast cell behaviour in coarse and binary packings: a reduction in $D_{\mathrm{F}}$ means that the micro-particle undergoing separation recognises the pore space as anisotropic. Therefore, in the model given by Eq. (1), the combination of both topology and anisotropy makes $B$ higher than unity.

\subsection{Bacillus}

In order to explain the observed behaviours of rod-like bacillus in coarse and binary packings, let us first consider the capillary HDC.

Capillary HDC separation of fibres from micro-particles was performed by Pascale and Maurice [53]. The classification was performed in a tube $12 \mathrm{~m}$ long and $15 \mathrm{~mm}$ in diameter. Three types of suspension were investigated: $3 \mu \mathrm{m} \mathrm{CaCO}_{3}$ particles, non-classified coniferous celluloses fibres (crude bleached pulp) of a length between 0.2 and $4 \mathrm{~mm}$, and a mixture of particles and fibres. It was found that the peak for fibres is situated ahead of the one for the particles and also ahead of the one for the flow. Particles have essentially the same time of passage as the carrier fluid. Moreover, longer fibres leave the column before the shorter ones.

In shear flow, a rod spends most of its flowing time positioned in the same direction as the streamlines, and therefore it has less effect on the flow, as mentioned by Jeffrey and Acrivos [54]. Consequently, the HDC separation is performed according to fibre diameter, and longer fibres had larger diameter. According to Rymsza [55], fibres $1.2-3.7 \mathrm{~mm}$ long have a $25-38 \mu \mathrm{m}$ diameter, which is significantly larger than the $\mathrm{CaCO}_{3}$ particles size.

Another effect might take place in rods flowing through porous media. The motion of a rod-like particle in extensional flow is quite different from the one observed in simple shear flow [56]. In extensional flow, a rod rotates until its longer axis coincides with the principal axis of extension [54].

For protein separation on a Sephadex column G-200, Siegel and Monty [57] showed that the order of elution of proteins from the column correlates well with the Stokes radius and does not depend fundamentally upon the molecular weight (see behaviour of ferritin and fibrinogen in Table 2). Fibrinogen has a completely different rod-like shape with axes ratio 20 (by viscosity) to 29 (by diffusion) [58] and diameter $\sim 5 \mu \mathrm{m}$ [59]. The fibrinogen distribution coefficient is extremely low and for Sephadex G-200 interparticle pores $\lambda<0.001$. The molecular rotational
Table 2

Molecular parameters of standard proteins separated on column of Sephadex G-200 [57]

\begin{tabular}{lclcl}
\hline Species & $\begin{array}{l}\text { Stokes radius } \\
(\mathrm{nm})\end{array}$ & $\begin{array}{l}\text { Molecular } \\
\text { weight }\end{array}$ & $\begin{array}{l}\text { Elution volume } \\
(\mathrm{mL})\end{array}$ & $\begin{array}{l}\text { Distribution } \\
\text { coefficient }\end{array}$ \\
\hline Fibrinogen & 10.7 & 330,000 & 88 & 0.03 \\
Ferritin & 7.9 & $1,300,000$ & 109 & 0.11 \\
\hline
\end{tabular}

effect may therefore be a reason for the observation reported by Siegel and Monty [57].

As seen from the coarse bed data, Fig. 8, elution volumes of yeasts (diameter $\sim 5 \mu \mathrm{m}$ ) and bacilli (length $\sim 5 \mu \mathrm{m}$ ) are close to each other, meaning that a rotational effect for the bacillus rod-like particle is present. This is the main advantage of porous media flow from capillary; owing to complex micro-pore topology, stream perturbations do not allow rod-like micro-particles to assume a more favourable position, parallel to the stream lines.

The above reasoning is valid for $\lambda$ around 0.01 (coarse particles packing) if it is assumed that for bacilli the effective diameter is equal to their length. However, when $\lambda$ approaches 0.1 (which is the case for binary packing) (Fig. 9), yeasts and bacilli retention are different. Moreover, the elution positions of yeasts and bacilli become inverted.

Theoretically, the retardation of bacilli could be the result of the attachment (adhesion)-detachment effect in the porous media. However, the absence of a tail on the bacilli breakthrough curve excludes this hypothesis. Hence, the interference of rodlike particles with the micro-pore topology is the more probable explanation for the observed retardation.

Moreover, by comparing curves 3 and $3^{\prime}$, it is possible to assume that rods, due to their similarity to a one-dimensional object ( $\lambda$ is defined as the ratio of length to the pore size), recognise the pore channel as a three-dimensional space, whereas, in the case of yeasts, pore channels are seen as a two-dimensional space.

This different interaction with the pore space topology may be supported by the following examples: in a parallelplate channel flow: $F(\lambda) \sim 1 /(1-\lambda)$ [60]; for a cylindrical pore $F(\lambda) \sim 1 /(1-\lambda)^{2}$, and for a 3D pore $F(\lambda) \sim 1 /(1-\lambda)^{3}$. Furthermore, Wood and Gladden [61] proposed the function $F(\lambda)=(1-\lambda)^{z}$, where they used $z=3.5$ and even 4.0.

The exclusion model for non-Brownian particles based on the particle size ratio to the pore size can be formulated in general form as [from Eq. (7) at pure exclusion effect when $F_{2}(\lambda)=0$ ]

$R=\frac{A}{(1-\lambda)^{z}}$

where $A$ is the coefficient proportional to the tortuosity factor and parameter $z=1,2$ or 3 depends on the spatial recognition by moving particles of the channel space.

In Fig. 9, two modes of exclusion were compared: $z=2$, curve 3 and $z=3$, curve $3^{\prime}$. The best-fit was obtained for $z=3$.

It is important to underline that the distortion effect generated by channel curvature yielded by bends increases the importance of both the length and diameter of a rod. The rod undergoes translational and rotational motions meaning that both geomet- 


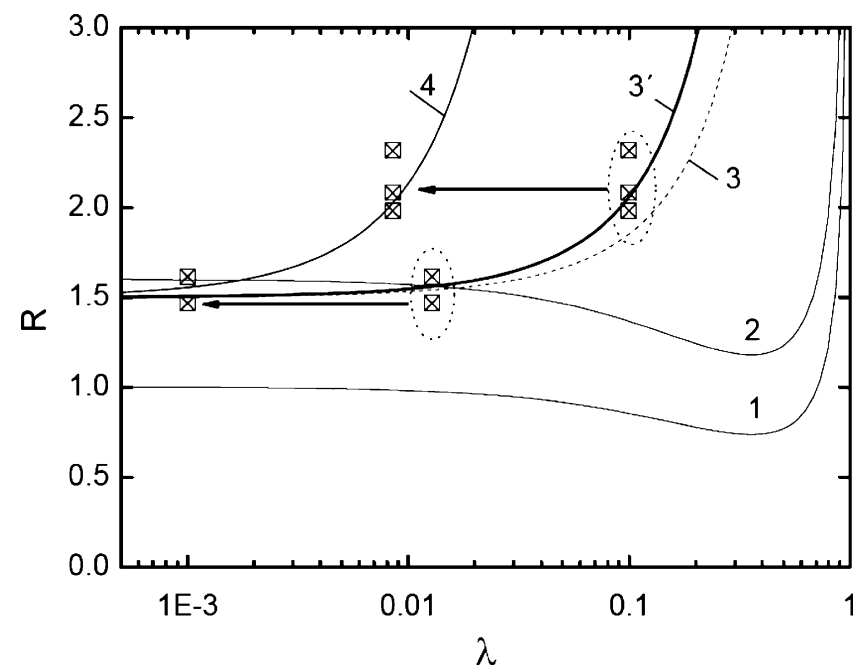

Fig. 11.

rical characteristics participate in the movement throughout the channel space. The rod diameter defines the rod's surface area and, hence, the friction force responsible for the relaxation phenomenon in $D e$ number. The rod length characterises the spatial volume of the channel $\lambda$, which is confirmed by Fig. 11 .

Based on the bends model proposed in Eq. (4), let us estimate the experimental conditions of binary and coarse packings to achieve better resolution.

Binary packing for $h=0.15 \mathrm{~m}$ gives bends number $n_{\text {b(binary) }}=2825$. The coarse packing, with the same amount of beads as the binary packing has $n_{\mathrm{b} \text { (coarse) }}=193$ that is almost 15 times less than that for the binary bed.

According to Eq. (3), the time $\theta_{\mathrm{p}}$ during which the rod-like bacillus is exposed to the distortion effect while crossing the bend depends on the flow velocity, and is $\theta_{\mathrm{p}} \approx 0.064 \mathrm{~s}$ for the binary packing and $1.08 \mathrm{~s}$ for the coarse packing. It must be admitted that the rotational relaxation time $\theta$ of rod-like particle in the complex geometry of packed bed is difficult to measure directly or to estimate. Therefore, only rough estimations can be made.

If we take $D e=0.5$ (where relaxation effect becomes significant), then by (3) the $\theta$ values are, for binary packing $0.031 \mathrm{~s}$ and for coarse packing $0.54 \mathrm{~s}$. The rotational relaxation time for xanthan [12], a stiff polymer with a nominal molecular weight $2 \times 10^{6}$, was measured to be $\theta=0.014 \mathrm{~s}$, which is in the scale of relaxation time for the binary packing used in the current experimentation.

Hence, assuming $\theta=0.031 \mathrm{~s}$, the recalculated Deborah number of coarse packing becomes $D e \approx 0.03$ that is far from $D e=0.5$ and the flow effect on the relaxation phenomena may be considered negligible.

\section{Conclusion}

The theoretical estimations made on the assumption of the effect of tortuosity and channel bends on the retardation effect for rigid rod-like particles were confirmed experimentally. The effect of pore topology plays an important role in the separation of particles of different shapes when the aspect ratio approaches $\lambda=0.1$. The complexity of the observed retardation phenomenon needs further investigation in this area.

It is plausible to presume that, to increase the impact of microtopology on micro-particle flow through a porous medium, we must increase (contrary to HPLC) the overall tortuosity by increasing the number of bends (thereby the micro-scale tortuosity) and the flow velocity (thereby limiting the relaxation time). By reducing the bend scale in comparison with the particle size, the opposite effect may be expected: bends convert into simple channel surface roughness. Moreover, for particles of a few microns in size, the gravitational force might contribute to retardation by affecting the particle's trajectory.

Further investigation is needed to clarify the particle-pore topology mechanism of the interference hereby identified. The obtained results may open interesting possible applications for bio-separation, for deep bed filtration, and to understand the motion of micro-particles (for instance viruses) in porous media.

\section{Acknowledgements}

The authors wish to thank FCT for the grant provided to Dr Alexander Yelshin. This work was developed under the framework of the project POCI_EQU_58337/2004, partially funded by FEDER.

\section{References}

[1] S. Bengtsson, L. Philipson, Biochim. Biophys. Acta 79 (1964) 399

[2] M.A. Scholl, R.W. Harvey, Environ. Sci. Technol. 26 (1992) 1410.

[3] R.W. Harvey, N.E. KInner, D. MacDonald, D. Metge, A. Bunn, Water Resour. Res. 29 (1993) 2713.

[4] R. Kretzschmar, K. Barmettler, D. Grolimund, Y.D. Yan, M. Borkovec, H. Sticher, Water Resour. Res. 33 (1997) 1129.

[5] L.L.C. Rehmann, C. Welty, R.W. Harvey, Water Resour. Res. 35 (1999) 987.

[6] R.W. Harvey, H. Harms, in: G. Bitton, et al. (Eds.), Encyclopedia of Environmental Microbiology, John Wiley \& Sons, New York, 2002, p. 3194.

[7] G. Stegeman, J.C. Kraak, H. Poppe, R. Tijssen, J. Chromatogr. A 657 (1993) 283.

[8] G. Stegeman, J.C. Kraak, H. Poppe, J. Chromatogr. A 634 (1993) 149.

[9] E. Venema, J.C. Kraak, H. Poppe, R. Tijssen, J. Chromatogr. A 740 (1996) 159.

[10] E.A. DiMarzio, C.M. Guttman, Macromolecules 3 (1970) 131.

[11] D.J. Nagy, C.A. Silebi, A.J. McHugh, J. Appl. Polym. Sci. 26 (1981) 1555.

[12] D.A. Hoagland, R.K. Prud'homme, Macromolecules 22 (1989) 775.

[13] M.T. Blom, E. Chmela, R.E. Oosterbroek, R. Tijssen, A. van den Berg, Anal. Chem. 75 (2003) 6761.

[14] A. Williams, E. Varela, E. Meehan, K. Tribe, Int. J. Pharm. 242 (2002) 295.

[15] E. Venema, R. de Leeuw, J.C. Kraak, H. Poppe, R. Tijssen, J. Chromatogr. A 765 (1997) 135.

[16] L. Lebrun, G.-A. Junter, J. Membr. Sci. 88 (1994) 253

[17] K.H. Hamaker, M.R. Ladisch, Sep. Purif. Methods 25 (1996) 47.

[18] M. Mota, J.A. Teixeira, A. Yelshin, Biotechnol. Progr. 17 (2001) 860.

[19] M.T. Blom, E. Chmela, J.G.E. Gardeniers, R. Tijssen, M. Elwenspoek, A. Van der Berg, Sens. Actuators B 82 (2002) 111.

[20] E.P.C. Mes, W.Th. Kok, H. Poppe, R. Tijssen, J. Polym. Sci., Part B: Polym. Phys. 37 (1999) 593.

[21] R. Tijssen, J. Bos, M.E. Kreveld, Anal. Chem. 58 (1986) 3036.

[22] Y.C. Guillaume, M. Thomassin, C. Guinchard, J. Chromatogr. Sci. 39 (2001) 361 . 
[23] E. Peyrin, Y.C. Guillaume, C. Garrel, A. Ravel, A. Villet, C. Grosset, J. Alary, A. Favier, Talanta 52 (2000) 1105.

[24] E. Peyrin, Y.C. Guillaume, C. Grosset, A. Ravel, A. Villet, C. Garrel, J. Alary, A. Favier, J. Chromatogr. A 886 (2000) 1.

[25] E. Peyrin, C. Caron, C. Garrel, A. Ravel, A. Villet, C. Grosset, A. Favier, Talanta 55 (2001) 291.

[26] F.X. Perrin, C.C. Masuyer, T.-T. Truong, C. Guinchard, J. Millet, J.P. Chaumont, M. Thomassin, Y.C. Guillaume, L. Nicod, J. Chromatogr. A 950 (2002) 281.

[27] C. André, Y.C. Guillaume, Chromatographia 59 (2004) 487.

[28] J. Hirabayashi, K. Kasai, J. Chromatogr. A 722 (1996) 135.

[29] J. Hirabayashi, K. Kasai, J. Chromatogr. A 893 (2000) 115.

[30] B.M. Rush, K.D. Dorfman, H. Brenner, S. Kim, Ind. Eng. Chem. Res. 41 (2002) 4652.

[31] B. Baidya, T. Mukherjee, J.F. Hoburg, Technical Proceedings of the International Conference on Modeling and Simulation of Microsystems, NanoTech 2002-MSM 2002, 22-25 April 2002, Puerto Rico, USA, 2002, p. 1.

[32] C. Bernhardt, Particle Size Analysis. Classification and Sedimentation Methods, Chapman and Hall, London, 1994.

[33] J.G. Savins, Ind. Eng. Chem. 61 (1969) 18.

[34] M. Mota, J.A. Teixeira, A. Yelshin, in: F. de Azevedo, E. Ferreira, K. Luben, P. Osseweijer (Eds.), Proceedings of 2nd European Symposium on Biochemical Engineering Science, 16-19 September 1998, Porto, University of Porto, Porto, Portugal, 1998, p. 93.

[35] M. Mota, J.A. Teixeira, A. Yelshin, in: M. Mota, E. Ferreira (Eds.), IV Iberian Congress on Biotechnology, I Iberio-American Meeting on Biotechnology, BIOTEC-98, 12-15 July 1998, Book of Abstracts, Barbosa \& Xaveir, Lda, Braga, Portugal, 1998, p. 211.

[36] M. Mota, J.A. Teixeira, R. Bowen, A. Yelshin, Proceedings of 8-th World Filtration Congress, 3-7 April 2000, Filtration Society, Brighton, UK, 2000, p. 57.

[37] M. Mota, J.A. Teixeira, A. Yelshin, Trans. Filtr. Soc. 1 (2001) 101.
[38] J.C. Kraak, Pure Appl. Chem. 69 (1997) 157.

[39] S. Budavari (Ed.), The Merck Index, An Encyclopedia of Chemicals, Drugs and Biologicals, Merck and Co, Rahway, USA, 1989.

[40] R. Dias, J.A. Teixeira, M. Mota, A. Yelshin, Sep. Purif. Technol. 37 (2004) 69.

[41] M. Auset, A. Keller, Water Resour. Res. 40 (2004) 1.

[42] A. Cernanský, Int. Chem. Eng. 29 (1989) 182.

[43] I. Ippolito, L. Samson, S. Bourlès, J.-P. Hulin, Eur. Phys. J. E3 (2000) 227.

[44] R.P. Beverly, Fluid/Part. Sep. J. 6 (1992) 27.

[45] D.J. Nagy, C.A. Silebi, A.J. McHugh, in: R.M. Fitch (Ed.), Polymer Colloids II, Plenum Publishing Corp, 1980, p. 121.

[46] A.J. McHugh, D.J. Nagy, C.A. Silebi, in: T. Provder (Ed.), Size Exclusion Chromatography, ACS Symp. Ser. ACS, 1980, p. 1.

[47] C.A. Silebi, A.J. McHugh, J. Colloid Interface Sci. 79 (1981) 264.

[48] A.J. McHugh, Crit. Rev. Anal. Chem. 15 (1985) 63.

[49] D.C. Francis, A.J. McHugh, in: T. Provder (Ed.), Size Exclusion Chromatography, ACS Symp. Ser. ACS, 1984, p. 3.

[50] R. Netrabukkana, K. Lourvanij, G.L. Rorrer, Ind. Eng. Chem. Res. 35 (1996) 458.

[51] C.N. Satterfield, Mass Transfer in Heterogeneous Catalysis, M.I.T. Press, Cambridge, England, 1970

[52] D.S. Cannell, F. Rondelez, Macromolecules 13 (1980) 1599.

[53] E. Pascale, R. Maurice, Int. Chem. Eng. 26 (1986) 257.

[54] D.J. Jeffrey, A. Acrivos, AIChE J. 22 (1976) 417.

[55] T.A. Rymsza, http://www.visionpaper.com/speeches_papers/996fps.html, 1999.

[56] X.-J. Fan, N. Phan-Thien, R. Zheng, J. Non-Newtonian Fluid Mech. 90 (2000) 47.

[57] L.M. Siegel, K.J. Monty, Biochim. Biophys. Acta 112 (1966) 346.

[58] M.T. Brik, E.A.T. Sapuk, Ultrafiltration, Naukova dumka, Kiev, 1989.

[59] C.E. Hall, H.S. Slayter, J. Biophys. Biochem. Cytol. 5 (1959) 11.

[60] M. Elimelech, Sep. Technol. 4 (1994) 186.

[61] J. Wood, L.F. Gladden, Appl. Catal. A: Gen. 249 (2003) 241. 